\title{
Joint Probability of Density Functions of Energy-Lifetime Distributions for Neutrons and Gamma-Rays from ${ }^{252}$ Cf Source
}

\author{
Senada Avdić, Izet Gazdić, Beco Pehlivanović, Alma Osmanović
}

\begin{abstract}
In contrast to some traditional neutron detectors, liquid scintillators are suitable for measuring spontaneous fission produced on the nanosecond time-scales. The high multiplicity events in a short period of time are signatures for fissile materials. The neutron and gamma multiplicities have potential to enable extraction of the fissile material attributes. The simulations presented in this paper were done using the MCNPX-PoliMi radiation transport code based on the Monte Carlo method. It was demonstrated that the energy and time information depending on the number of neutrons and gamma-rays emitted in spontaneous fission events of ${ }^{252} \mathrm{Cf}$ contribute additionally to characterize a fission source.
\end{abstract}

Keywords: Monte Carlo method, fissile material, neutron and gamma multiplicities, joint probability density function.

\section{Introduction}

Neutron detection with liquid scintillators has been used successfully in recent applications in the areas of homeland security and nuclear nonproliferation [1]. Liquid organic scintillators such as EJ-309 are sensitive to both neutrons and gammas and have good pulse-shape discrimination properties [2].

Detection of special nuclear materials (SNM) is of high importance in nuclear safeguards. These materials emit simultaneously multiple neutrons and gamma-rays, through spontaneous or induced fission. Multiplicity and time-correlation are useful features to detect fission events and identify special nuclear materials [3].

Since there are no possibility in our laboratory for the experimental studies of SNM sources requiring authorized access and the use of the advanced experimental equipment, we were able to use complex numerical simulations to characterize a fission source. We

Manuscript received May 12, 2016; accepted December 27, 2016.

Senada Avdić, Izet Gazdić and Alma Osmanović are with the Department of Physics, Faculty of Science, University of Tuzla, Tuzla, Bosnia and Herzegovina; Beco Pehlivanović is with the Faculty of Educational Science, University of Bihac, Bihac, Bosnia and Herzegovina. 
have carried out the simulations of spontaneous fission (SF) of ${ }^{252} \mathrm{Cf}$ source using the MCNPX-PoliMi code based on the Monte Carlo (MC) method [4]. The model for spontaneous fission includes prompt neutron energy distributions that depend on the number of neutrons emitted in the individual fission events while gamma rays are sampled independently of the neutrons.

The joint probability of density function is related to a joint number of neutrons and/or gamma-rays emitted from fissile materials. Since more parameters can enable more possibilities for improvements in identifying the presence of SNM, the joint probability of density functions (JPDFs) for energy-lifetime distributions for neutrons and gamma-rays from ${ }^{252} \mathrm{Cf}$ source were analyzed, taking into account dependence on the different numbers of neutrons emitted in SF process. The goal of this paper was to investigate the feasibility of using the JPDFs of energy-lifetime distributions of neutrons and gamma-rays from a fission source to obtain additional information for recognition of SF source such as ${ }^{252} \mathrm{Cf}$.

The paper is organized as follows: in Section 2 The MCNPX-PoliMi neutron and gamma multiplicity modelling is described; the simulated results and discussion are presented in Section 3 and conclusions are given in Section 4.

\section{The MCNPX-PoliMi neutron and gamma multiplicity modelling}

The MCNPX-PoliMi code [4] is a modified version of the MCNPX ver. 2.7.0 code with many enhancements including the correlated particle detection of interest in several SNM applications. The code is used for detailed modelling of particle-nucleus interactions, and event-by-event detection analysis as well as for accurate description of complex geometries. In this work, the MCNP-PoliMi code was applied for the MC modelling of detailed detection physics taking into account time-correlated events. Detector response, including the time difference between correlated particles, was calculated using MPPost [5], a specialized post-processing script for converting the detection physics into the corresponding response.

A characteristic feature of SNM sources is that they emit correlated neutrons and gammarays with characteristic signatures. An average of 4 neutrons are promply emitted within approximately $4 \cdot 10^{-14} \mathrm{~s}$ following the scission event while an average of 7-8 gammarays are emitted promptly from ${ }^{252} \mathrm{Cf}$ source, within $1 \mathrm{~ns}$ from the fission event [6]. The MCNPX-PoliMi numerical code performs sampling of the number of prompt neutrons and gamma rays from appropriate distributions for each spontaneous fission event. The neutron and gamma-ray multiplicity distributions for the spontaneous fission of ${ }^{252} \mathrm{Cf}$ source are given in Fig.1.

Taking into account that the neutrons and gamma rays emitted from fission fragments compete for a part of the excitation energy that is available in a given scission event the energy spectrum of prompt neutrons is shifted toward smaller energies as the number of 


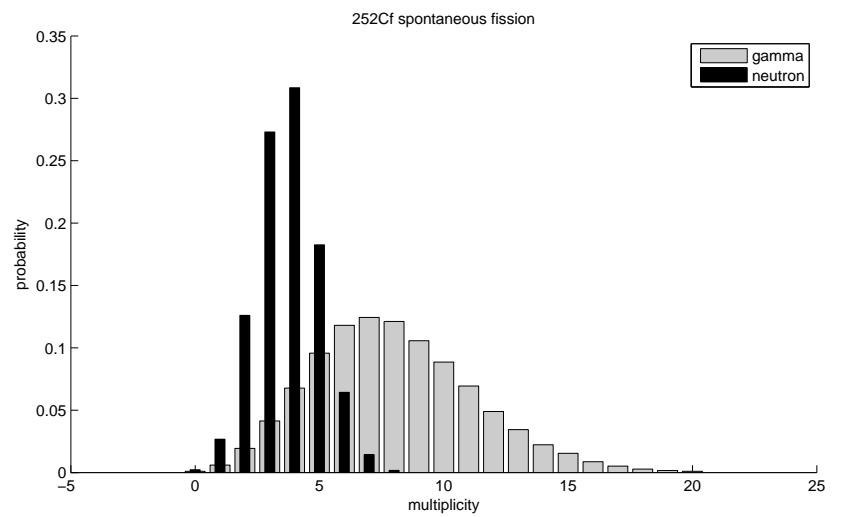

Fig. 1. Neutron and gamma-rays multiplicity distributions for the spontaneous fission of ${ }^{252} C f$.

emitted neutrons increases [4]. Fig. 2. shows the neutron energy distributions implemented in MCNPX-PoliMi for ${ }^{252} \mathrm{Cf}$ depending on the number of neutrons emitted in a fission event.

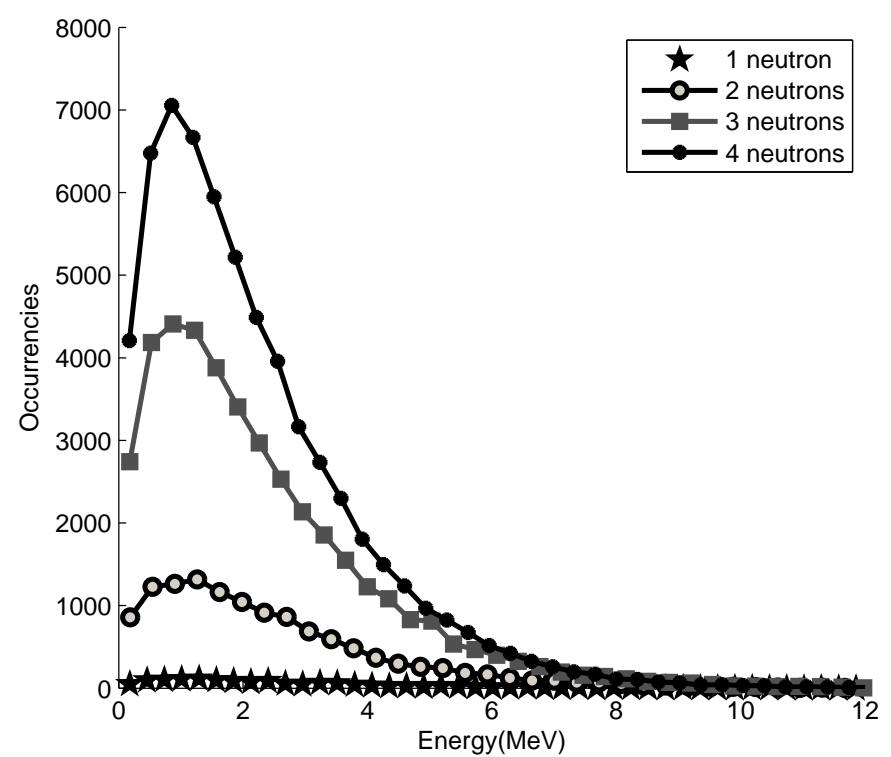

Fig. 2. Neutron energy distributions of ${ }^{252} C f$ for various multiplicities. 


\section{The simulated results and discussion}

In order to distinguish time-correlated particles induced by a non-fission source from those emitted by a fission source, the use of more parameters such as energy, lifetime, joint neutron-gamma-ray multiplicity, Feynman variance to mean ratio for neutrons and gamma photons [7], etc. can be significantly useful for identifying the presence of SNM. The statistical properties of a joint number of neutrons and gamma-rays emitted from fissile samples are described by the analytical approach [8]. It is well known that neutrons might be more favourable for measuring since the self-shielding effect for gamma photons increases in the case of large samples. However, the joint detection of neutrons and photons might lead to shorter measuring times and/or to get more data about the sample. Simulations of Joint Probability Density Functions (JPDFs) of lifetime and energy for neutrons and gamma-rays depending on the number of neutrons and gamma-rays emitted by a fis sion source such as ${ }^{252} \mathrm{Cf}$ are not well presented in the literature. It can be seen in Fig. 3. that the fraction of prompt neutrons and gamma photons from ${ }^{252} \mathrm{Cf}$ decreases with their lifetimes for various multiplicities.
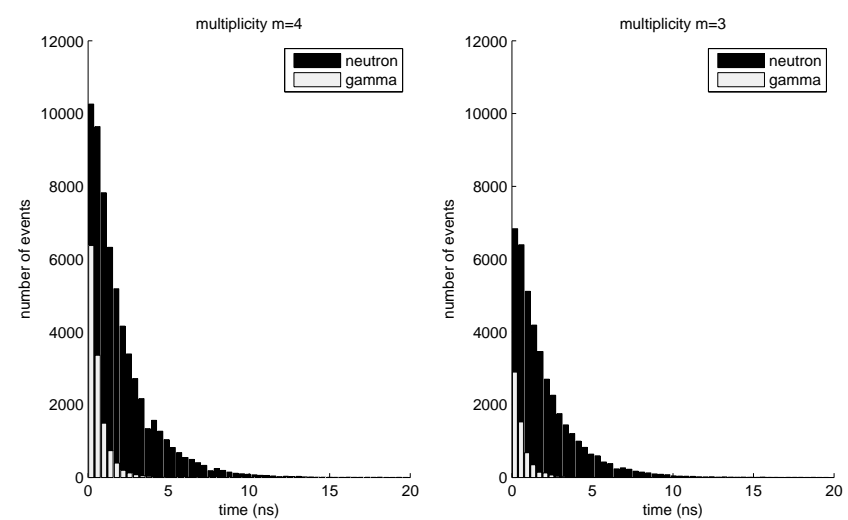

Fig. 3. The fraction of prompt neutrons and gamma photons from ${ }^{252} \mathrm{Cf}$.

The JPDF of energy-lifetime distribution for neutrons with multiplicity of 2 neutrons is given in Fig. 4. It can be seen that the largest fraction of neutrons is with energy of about 2 $\mathrm{MeV}$ and lifetime of about $2 \mathrm{~ns}$. The fraction of neutrons with higher energy and lifetimes decreases.

Fig. 5. shows the JPDF of energy-lifetime distribution for gamma photons and multiplicity of 2 gamma-rays. It can be seen that the largest fraction of gamma photons is with energy about $0.4 \mathrm{MeV}$ and lifetime of about $0.2 \mathrm{~ns}$. Similarly to neutrons, the fraction of gamma photons with higher energy and lifetimes decreases. 


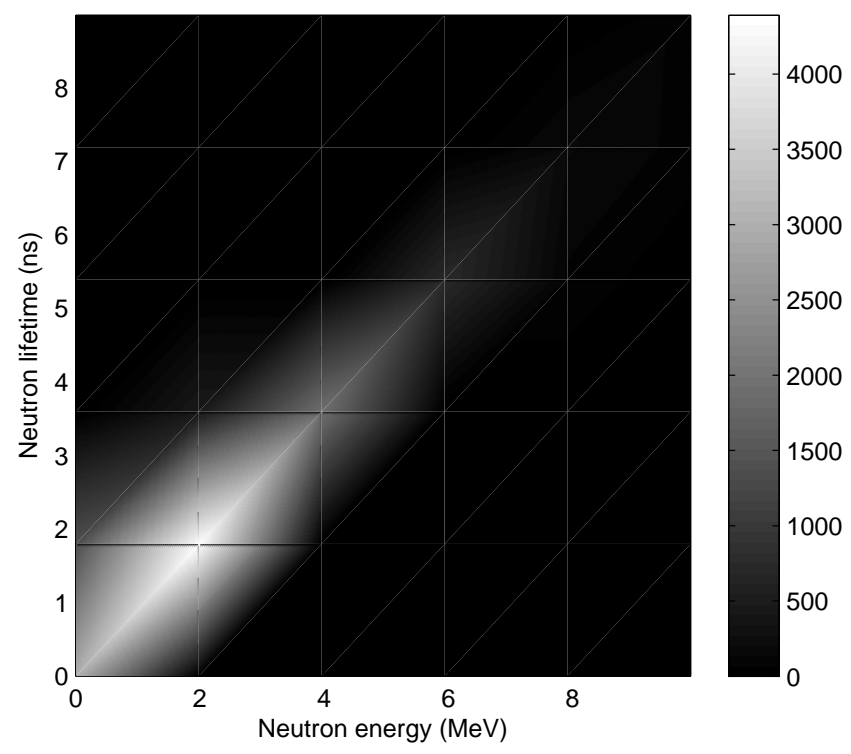

Fig. 4. The JPDF of energy-lifetime distribution for neutrons.

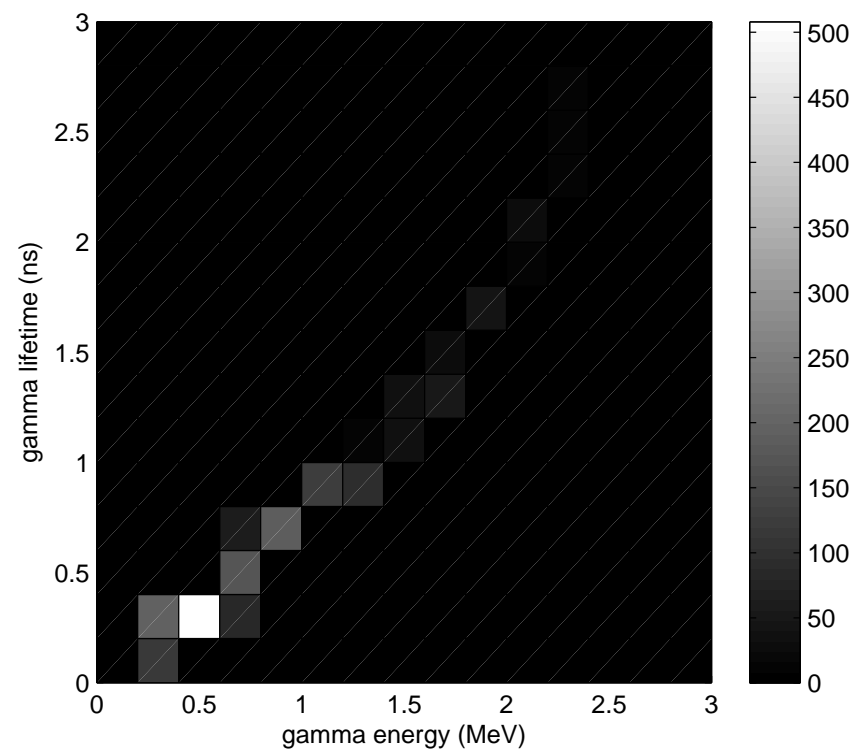

Fig. 5. The JPDF of energy-lifetime distribution for gamma photons. 


\section{Conclusions}

Liquid scintillators have been used widely for fast neutron detection and identification of special nuclear materials (SNM) in different nuclear safeguards applications. Since liquid scintillators are fast enough to detect the neutron and gamma multiplicities in a short period of time, it was numerically investigated a possibility to use the JPDFs to extract additional attributes useful to characterize fissile materials. The joint energy-lifetime distributions of neutrons and of gamma-rays emitted from ${ }^{252} \mathrm{Cf}$ spontaneous fission source were analyzed. It was demonstrated that the JPDFs including dependence on the number of neutrons and gamma-rays emitted in a fission event have potential to enable additional information on fissile material.

It was planned in the framework of future work to analyze the JPDF s for non-fission sources and hence to investigate a possibility to discriminate SNM source from a nonfission source.

\section{References}

[1] J. A. Mullens, J. D. Edwards, S. A. PozZI, Analysis of Scintillator Pulse-Height for Nuclear Material Identification, Institute of Nuclear Materials Management 45th Annual Meeting, Orlando, FL, 2004, July 18-22.

[2] Esposito, B., Kaschuck, Y., Rizzo, A., Bertalot, A., Pensa, A., 2004. Digital pulse shape discrimination in organic scintillators for fusion applications. Nuclear Instruments and Methods in Physics Research A 518, Issues 12, 626-628.

[3] G. Miloshevsky, A. Hassanein, Multiplicity correlation between neutrons and gammarays emitted from SNM and non-SNM sources, Nucl. Instrum. Methods Phys. Res., Sect. B 342 (2015) 277-285.

[4] S. A. POZZI ET AL., MCNPX-PoliMi for nuclear nonproliferation applications, Nucl. Instrum. Methods Phys. Res., Sect. A 694 (2012) 119-125.

[5] E. C. Miller, A. Poitrasson-Riviere, A. Enqvist, J. L. Dolan, S. Prasad, M. M. Bourne, K. Weinfurther, S. D. Clarke, M. Flaska, S. A. Pozzi, E. Padovani, J. K. Mattingly, "MCNPX-PoliMi Post-Processor (MPPost) Manual", $R S$ ICC package number C00791 MNYCP 00, www.rsicc.ornl.gov

[6] Robert Vandenbosch, John R. Huizenga, Nuclear Fission, Academic Press Inc., New York, 1973.

[7] D. Chernikova, K. Axell, S. Avdic, I. Pazsit, A. Nordlund, S. Allard, The neutron-gamma Feynman variance to mean approach: Gamma detection and total neutron -gamma detection (theory and practice), Nuclear Instruments and Methods in Physics Research A, Vol. 782, pp. 47-55, 2015.

[8] A. EnQVist, S. A. Pozzi, I. PAZSIT, The detection statistics of neutrons and photons emitted from a fissile sample, Nucl. Instrum. Methods Phys. Res., Sect. A 607 (2009) 451-457. 